\title{
Die Durchstarterin
}

CIMT BEI ICP Constraint-induced Movement Therapy ist bei Kindern mit Zerebralparese effektiv. Das wies Astrid Stahlmann in ihrer Bachelorarbeit nach. Nach dem Studium eröffnete die Ergotherapeutin eine eigene Praxis und schrieb sich direkt für einen Masterstudiengang ein. Langeweile ausgeschlossen.

Was war die größte Herausforderung während des Studiums?

Das Schreiben der Bachelorarbeit. Die Arbeit war sehr theoretisch und zeitaufwendig, ich musste viele Studien lesen.

Welchen Tipp würden Sie nachfolgenden Studenten geben?

Wer es zeitlich schafft, sollte nebenbei praktisch arbeiten. Für mich war das ein wertvoller Ausgleich zur Theorie. Außerdem kann man so Lerninhalte in der Praxis ausprobieren.

\section{Wohin würden Sie gerne einmal reisen?}

Ich bin ein absoluter Kreuzfahrt-Fan. Am liebsten würde ich einmal mit dem Schiff nach Australien, Asien oder Neuseeland reisen.

Was wünschen Sie sich für Ihre Zukunft?

Dass meine Praxis erfolgreich läuft, dass ich die Arbeit gut mit meinem anstehenden Masterstudium vereinbaren kann und dass ich trotzdem noch ein bisschen Zeit für mich habe. 


\section{Effektivität von CIMT bei Kindern mit Zerebralparese}

\section{Die Bachelorarbeit}

Die Constraint-induced Movement Therapy (CIMT) ist eine vielversprechende Therapiemethode in der neurologischen Rehabilitation. Bislang kennt man sie vor allem aus der Behandlung von Erwachsenen. Da sie den Gebrauch der betroffenen Extremität erzwingt und einen hohen Therapieintervall erfordert, ist sie für Kinder sehr anspruchsvoll. Darum hat es sich Astrid Stahlmann für ihre Bachelorarbeit zum Ziel gesetzt, herauszufinden, wie effektiv diese Therapiemethode kombiniert mit bimanuellem Training bei Kindern mit einer Zerebralparese ist.

Um dieser Frage nachzugehen, hat sie zunächst eine Literaturrecherche durchgeführt. Dabei durchsuchte sie die Datenbanken PubMed, Cochrane Library und PEDro. Lediglich zwei englischsprachige Studien aus dem Jahr 2010 entsprachen den festgelegten Einund Ausschlusskriterien. Deren Forscher analysierten insgesamt 68 Kinder mit hemiplegischer Zerebralparese. Um die Ergebnisse beider Studien statistisch erfassen zu können, führte Astrid Stahlmann anschließend eine Metaanalyse durch.

$\rightarrow$ Stahlmann A. Effektivität von Constraintinduced Movement Therapy bei Kindern mit Zerebralparese. Bachelorarbeit an der SRH Fachhochschule für Gesundheit Gera; 2011

\section{Ergebnisse}

Astrid Stahlmann fand heraus, dass ...

> die aktuelle Literatur zu CIMT in Kombination mit bimanuellem Training schwach aufgestellt ist: Es gibt zu wenige Studien mit zu geringen Teilnehmerzahlen.

> CIMT ein sehr effektives Verfahren zur Verbesserung der betroffenen Extremität bei Kindern mit Zerebralparese ist.

> Kinder ihre Belastungsgrenze deutlich frü- her als Erwachsene erreichen. Erwachsene können pro Tag etwa sechs Stunden CIMT absolvieren, Kinder hingegen gelangen nach ungefähr drei Stunden Therapie an ihre Grenze. Das liegt unter anderem an einer geringeren Aufmerksamkeits- und Konzentrationsspanne.

> bei Kindern mit Zerebralparese eine hochintensive CIMT besser wirkt als eine niedrig dosierte.

> CIMT die Grundlage dafür schafft, den betroffenen Arm in Bewegungen einzubeziehen.

> sich CIMT signifikant positiv auf die manuelle Geschicklichkeit, die Motorik sowie die Selbstständigkeit und Partizipation im Alltag auswirkt.

> bei CIMT die Intensität entscheidend für den Erfolg ist: je höher das Intervall, desto wahrscheinlicher ist ein positives Ergebnis.

$>$ ein zusätzliches bimanuelles Training die erlernten Fähigkeiten mit der Restriktion festigt.

\section{Fazit}

Zusammenfassend hält Astrid Stahlmann fest, dass ...

> es insgesamt mehr Studien mit höheren Teilnehmerzahlen braucht - auch mit negativem Effekt -, um den Gesamteffekt von CIMT mit anschließendem bimanuellem Training zu bestätigen.

$>$ es in einer ambulanten Ergotherapiepraxis kaum möglich ist, CIMT durchzuführen. Der zeitliche und der personelle Aufwand sind zu groß.

$>$ die Constraint-induced Movement Therapy in Verbindung mit bimanuellem Training bei Kindern mit Zerebralparese eine sehr wirksame Behandlungsmethode ist. Gegenüber normaler Therapie hat sie einen positiven Effekt auf die Funktionsebene.

Simone Gritsch 